\title{
Survival during influenza-associated bacterial superinfection improves following viral- and bacterial-specific monoclonal antibody treatment
}

\author{
Keven M. Robinson, ${ }^{1}$ Krishnaveni Ramanan, ${ }^{1}$ Joshua M. Tobin, ${ }^{1}$ Kara L. Nickolich, ${ }^{1}$ \\ Matthew J. Pilewski, ${ }^{1}$ Nicole L. Kallewaard, ${ }^{3}$ Bret R. Sellman, ${ }^{3}$ Taylor S. Cohen, ${ }^{3}$ and John F. Alcorn ${ }^{2}$ \\ 'Division of Pulmonary, Allergy, and Critical Care Medicine, Department of Medicine, University of Pittsburgh, Pittsburgh, \\ Pennsylvania, USA. ${ }^{2}$ Division of Pulmonary Medicine, Department of Pediatrics, University of Pittsburgh, Pittsburgh, \\ Pennsylvania, USA. ${ }^{3}$ Department of Microbial Sciences, Medlmmune, Gaithersburg, Maryland, USA.
}

\begin{abstract}
Postinfluenza bacterial superinfections cause increased morbidity and mortality compared with singular infection with influenza during both pandemics and seasonal epidemics. Vaccines and current treatments provide limited benefit, a rationale to conduct studies utilizing alternative therapies. FY1 and an optimized version, MEDI8852, anti-influenza HA mAbs, have been shown to neutralize influenza virus during singular influenza infection. MEDI4893*, an anti-Staphylococcus aureus $\alpha$-toxin $\mathrm{mAb}$, has been shown to improve survival when administered prophylactically prior to S. aureus pneumonia. Our objective was to determine if mAbs can improve survival during postinfluenza bacterial pneumonia. We administered FY1 in a murine model of postinfluenza methicillin-resistant $S$. aureus (MRSA) pneumonia and observed improved survival rates when given early during the course of influenza infection. Our findings indicate decreased lung injury and increased uptake and binding of bacteria by macrophages in the mice that received FY1 earlier in the course of influenza infection, corresponding to decreased bacterial burden. We also observed improved survival when mice were treated with a combination of FY1 and MEDI4893* late during the course of postinfluenza MRSA pneumonia. In conclusion, both FY1 and MEDI4893* prolong survival when used in a murine model of postinfluenza MRSA pneumonia, suggesting pathogenspecific $m A$ bs as a possible therapeutic in the context of bacterial superinfection.
\end{abstract}

Conflict of interest: JFA received research funding from Medlmmune to conduct these studies. NLK, TSC, and BRS are employees of Medlmmune.

Copyright: () 2019 American Society for Clinical Investigation

Submitted: October 15, 2018

Accepted: June 5, 2019

Published: July 25, 2019.

Reference information: /CI Insight. 2019;4(14):e125554. https://doi. org/10.1172/ji.insight.125554

\section{Introduction}

Influenza virus infection is a serious public health threat. Annually, epidemics result in 3-5 million severe influenza cases and an estimated 300,000-500,000 deaths worldwide from influenza-related respiratory disease (1). Seasonal influenza epidemics are caused by 2 types of influenza viruses: A and B. Influenza A viruses are classified into subtypes based on their surface glycoproteins HA and neuraminidase (NA). Only 3 combinations are known to have widely circulated in humans: H1N1, H2N2, and H3N2. Currently, A/ $\mathrm{H} 1 \mathrm{~N} 1$ and $\mathrm{A} / \mathrm{H} 3 \mathrm{~N} 2$ cause seasonal epidemics (2). Viruses containing H5, H7, H9, and H10 HAs cause sporadic human infections related to transmission directly from avian species (3). Influenza virus genomes include 8 gene segments, which can evolve over time accruing mutations, known as antigen drift, or can undergo genome reassortment, known as antigenic shift. Both antigenic drift and antigenic shift can lead to new antigenic variants that escape current vaccines and have the potential to cause influenza pandemics (2). Influenza virus pandemics have both devastating human and economic consequences.

Influenza infection can be complicated by secondary superinfections such as bacterial pneumonia. Bacterial superinfection leads to increased morbidity and mortality, and an important part of pandemic prevention and management is understanding the relationship between influenza infection and secondary bacterial infection (4-8). Throughout the 1918 influenza A H1N1 pandemic, more than 50 million people died from influenza complicated by secondary bacterial pneumonia (7). During the 2009 influenza A H1N1 pandemic, $25 \%-50 \%$ of hospitalized patients infected with influenza also had a bacterial superinfection $(4,5)$. Among influenza-associated pediatric deaths in the United States between 2004 
and 2012, approximately $40 \%$ of children were infected with both influenza and a bacterial organism. Most often, secondary bacterial pneumonia is caused by either Staphylococcus aureus or Streptococcus pneumoniae $(9,10)$. Methicillin-resistant $S$. aureus (MRSA) clonotypes USA300 and USA400 are most likely to cause secondary pneumonia following influenza $(11,12)$. $S$. aureus has multiple virulence factors that are utilized to evade immune host defense. Influenza infection dysregulates both innate and adaptive immune responses to bacterial pneumonia, predisposing infected individuals to secondary bacterial superinfection (13). Influenza infection also causes epithelial cell damage within the respiratory tract and allows for increased areas for bacteria to attach (11). Currently, available vaccines and therapies for influenza infection and influenza complicated by a secondary bacterial infection have limitations, in efficacy and timing of treatment, and new therapeutic development is a key to future pandemic prevention.

HA is composed of a globular head domain that binds to sialic acid receptors on host cells and a stem domain that allows for fusion between viral and cellular membranes. The globular head domain is the primary target of vaccination- or infection-induced antibodies that block sialic acid receptor binding. This method of vaccination is problematic due to the accumulation of amino acid mutations within the head domain that necessitate annual development of new influenza vaccines. Conversely, the HA stem domain is highly conserved between subtypes and provides a target for multisubtype antibody protection. Recently, mAb FY1 was identified and further optimized to create MEDI8852. These antibodies bind to the HA stem and broadly neutralize influenza viruses (3). Furthermore, an anti-S. aureus $\alpha$-toxin mAb (LC10, also known as MEDI4893*) was identified and was shown to decrease bacterial burden and improve survival during $S$. aureus pneumonia in mice $(14,15)$. MEDI8852 and MEDI4893 (suvratoxumab) are currently in clinical testing for the treatment of influenza and prevention of pneumonia in intubated patients in the intensive care unit, respectively (https://www.clinicaltrials.gov/). Our current work explores the activity of FY1 and MEDI4893* during influenza complicated by secondary bacterial superinfection.

\section{Results}

Influenza HA targeting mAb FY1 decreases acute lung injury and protects against influenza-associated bacterial pneumonia. Mice infected with influenza and then challenged with MRSA to mimic human influenza-associated bacterial superinfection have increased bacterial burden, inflammation, and mortality compared with those infected with $S$. aureus or influenza alone $(16,17)$. Prior studies have demonstrated that the anti-influenza HA mAb MEDI8852 and parent FY1 inhibit influenza virus replication and protect against morbidity and mortality during influenza infection, in both in vitro and in vivo murine models (3). To examine the effect of FY1 during influenza A and MRSA superinfection, C57BL/6 mice were challenged with influenza A PR/8/34 and then infected 6 days later with MRSA (clonotype USA300). FY1 was administered at different time points, days 1-5, after influenza infection and the control group received IgG isotype control on day 1 after influenza infection (Figure 1A). Mice that received FY1 on days 1 and 2 after influenza infection had decreased bacterial burden (Figure 1B) and weight loss (Figure 1, C and D) compared with mice that did not receive the antibody. Interestingly, mice that received FY1 on days 3, 4, or 5 after influenza infection had reduced bacterial burden without protection against influenza-induced weight loss. In order to determine if FY1 improves survival in addition to bacterial clearance, we treated mice with the anti-HA mAb on days 1,3 , and 5 after influenza and infected with MRSA on day 6 after influenza. Mice that received the anti-HA antibody on days 1 or 3 after influenza infection had significantly increased survival compared with control mice and those treated with the antibody on day 5 (Figure 1E). Notably, singular infection with either influenza or MRSA alone is not lethal (data not shown). Next, we measured total protein levels and IgM levels in bronchoalveolar lavage fluid (BALF) to assess permeability of the alveolar-capillary membrane. Mice that received FY1 at earlier time points had decreased protein and IgM in the BALF (Figure 1, F and G), demonstrating decreased alveolar-capillary membrane and acute lung injury at these time points. During influenza and bacterial superinfection, influenza burden is increased compared with mice that are infected with influenza alone (16). In our current experiments, mice that received FY1 earlier in the course of influenza infection had decreased viral burden at 6 days after infection (Figure 2A). Influenza is known to elicit a strong IFN response including upregulation of IFN- $\beta,-\lambda$, and $-\gamma$, and IFN signaling. In particular, type I and type III IFN have been linked to impaired antibacterial immune responses $(18,19)$. IFN- $\beta,-\alpha$, and $-\lambda$ (Figure 2, B-D) were significantly decreased in the mice that received the antibody earlier in the course of infection, corresponding to viral titers. Although IFN- $\gamma$ trended to be less in the antibody-treated mice, there were no statistically significant changes between groups (Figure 2E). These data suggest that FY1 provides benefit in part by limiting viral replication and subsequent IFN responses in the lung. 
Influenza $H A$ targeting $m A b$ decreases airway inflammation and inhibits cytokine/chemokine responses in the lung during influenza-associated bacterial pneumonia. Preceding influenza infection leads to alteration of the innate immune response to secondary bacterial pneumonia. Influenza depletes up to $90 \%$ of mouse resident alveolar macrophages by 1 week after infection, and recruited inflammatory monocytes replace the resident cells during bacterial superinfection (20). Previous studies using different types of bacteria as a secondary challenge and assessing different time points have all shown enhanced neutrophil recruitment at days 3-7 after influenza infection when mice are challenged with a secondary bacterial pneumonia compared with influenza or bacterial infection alone $(17,21)$. To investigate the effect of FY1 on the inflammatory response during influenza A and MRSA superinfection, we first analyzed immune cells and cytokines in the BALF. We found decreased numbers of multiple immune cell types that appeared morphologically similar to neutrophils, macrophages, and lymphocytes in the airway compartment of mice that received the antibody compared with control mice by cytospin differential counting (Figure 3, A-D). In addition, there was suppression of both inflammatory and antiinflammatory cytokines in the lungs of mice that received the mAb, including IL- 6 and IL-10 (Figure 3, E and G), and decreased amounts of chemokines keratinocyte chemoattractant [KC] (Figure 3F) MCP-1, MIP- $1 \alpha$, MIP-1 $\beta$, RANTES, and GM-CSF (Supplemental Figure 1; supplemental material available online with this article; https://doi.org/10.1172/jci.insight.125554DS1). Notably, not all chemokines and cytokines were altered in mice that received the mAb. Our laboratory has previously studied both type 2 and type 17 immunity during influenza complicated by MRSA pneumonia and with the exception of IL-33, none of the cytokines central to type 2 or type 17 immune responses was altered in response to the influenza $\mathrm{mAb}$ (Supplemental Figures 2 and 3). These data demonstrate the ability of FY1 to suppress pulmonary inflammation and acute lung injury when delivered several days following influenza infection.

Influenza $H A$ targeting $m A$ bs alters macrophage and neutrophil populations in the lung during influenza-associated bacterial pneumonia and enhances the ability of macrophages to bind and uptake bacteria. As previously mentioned, we found decreased numbers of cells that appeared morphologically similar to neutrophils in the airways of mice that received the antibody compared with control mice by performing cytospin differentials on BALF. To further dissect the effects of FY1 on cell populations in the lung during influenza and MRSA superinfection, we performed a detailed analysis of the monocyte and granulocyte populations in the lung by flow cytometry. There was an increased percentage of $\mathrm{Cd}_{11 \mathrm{~b}^{+}}$cells, CD $11 \mathrm{c}^{+}$ cells, and $\mathrm{Cd} 11 \mathrm{~b}^{+} \mathrm{CD} 11 \mathrm{c}^{+}$dendritic cells in mice that received the antibody early during the course of influenza infection (Figure 4, A-C). In contrast, there was a decreased percentage of $\mathrm{F} 480^{+}$cells in the mice that received the antibody, including total $\mathrm{F}_{4} 80^{+}$cells, $\mathrm{F} 480^{+}$SiglecF ${ }^{+} \mathrm{CD}_{11} \mathrm{c}^{+}$alveolar macrophages, and $\mathrm{F}_{480}{ }^{+}$SiglecF $\mathrm{F}^{-} \mathrm{CD} 11 \mathrm{c}^{-}$interstitial macrophages (Figure 4, D-F), and a decreased percentage

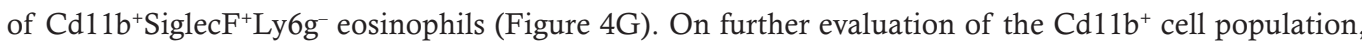
there was an increased percentage of cells dual-positive for both Cd11b and Ly6g (neutrophils) in the mouse group that received the antibody early in the course of infection (Figure $4 \mathrm{H}$ ). Furthermore, within the $\mathrm{CD} 11 \mathrm{~b}^{+} \mathrm{Ly}_{6 \mathrm{~g}}{ }^{+}$population, there was a higher percentage of $\mathrm{Ly} \mathrm{g}^{\text {thi }}$ cells, mature neutrophils, in the mouse group that received the antibody early in the course of infection (Figure 4I). To investigate if neutrophils are required for FY1-mediated protection, C57BL/ 6 mice were challenged with influenza A PR/8/34 and then infected 6 days later with MRSA (USA300). FY1 was administered on day 1 after influenza infection. Mice received $250 \mu \mathrm{g} 1 \mathrm{~A} 8$ (anti-Ly6G) or IgG control by i.p. injection at 48, 24 , and 2 hours prior to $S$. aureus challenge. Although we have previously used this methodology to measure neutrophil depletion (22), we performed flow cytometry to ensure neutrophils were adequately depleted within the lung (data not shown). Bacterial burden was unchanged between the mouse groups that received FY1 with and without the neutrophil depletion antibody (Figure 5A), demonstrating that neutrophils are not required for FY1-mediated protection. To further investigate the role of phagocytic cells in our model, C57BL/6 mice were challenged with influenza A PR/8/34 and then infected 6 days later with FITC-labeled MRSA (USA300). FY1 and an isotype control were administered on day 1 after influenza infection. Examination of the $S$. aureus ${ }^{+}$neutrophils revealed no difference in the total number of FITC $^{+}$cells in mice that received FY1 compared with control mice (Figure 5B). Examination of the $S$. aureus $^{+}$macrophages, both alveolar and interstitial, revealed an increase in the total number of FITC ${ }^{+}$ cells in mice that received FY1 compared with control mice (Figure 5, C and D). These data suggest that although FY1 inhibits the percentage of macrophages in the lung, it enhances the ability of both alveolar and interstitial macrophages to phagocytose $S$. aureus. 
A

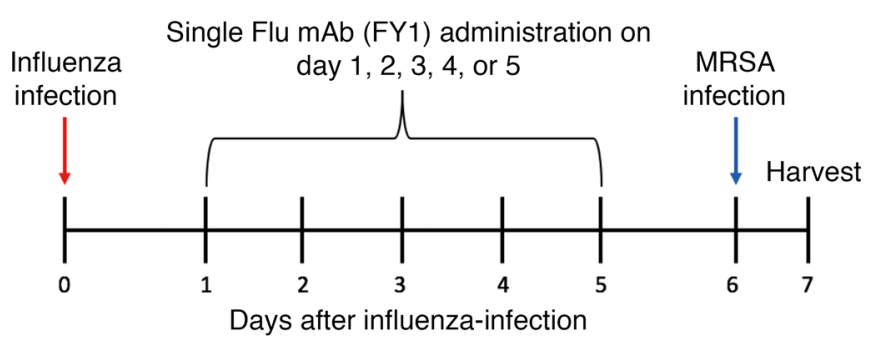

C

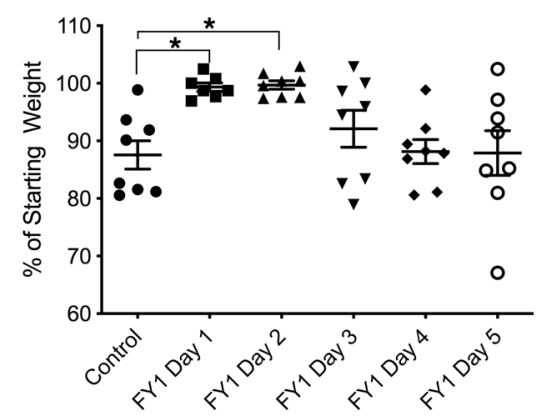

E

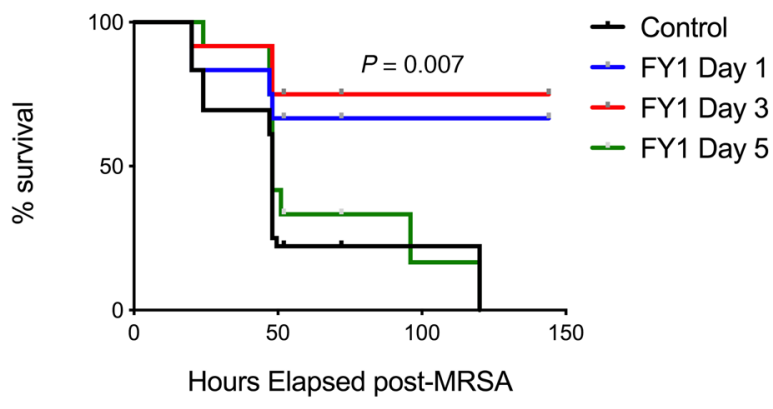

G

$\lg M$

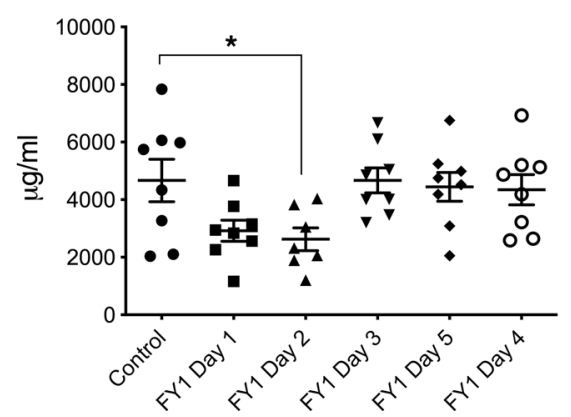

B

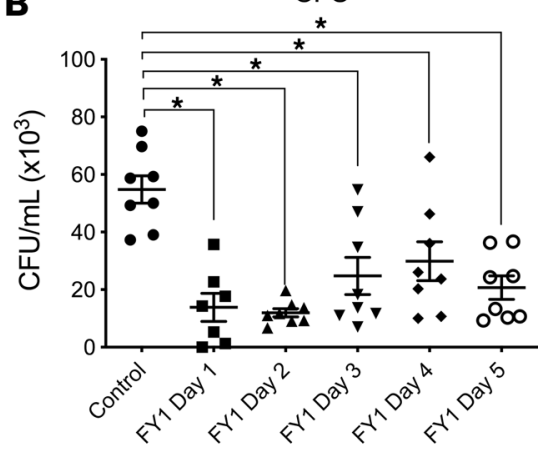

D

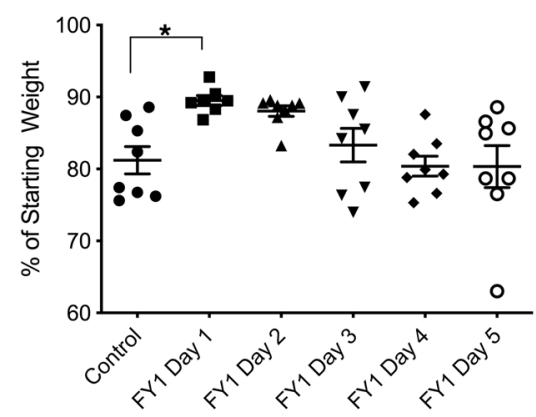

$\mathbf{F}$

Protein

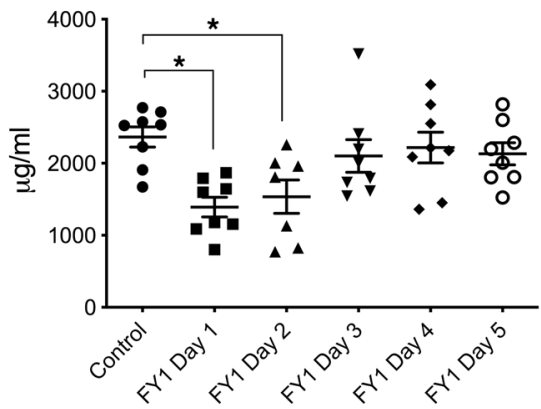

Figure 1. Influenza HA targeting mAb FY1 decreases acute lung injury and protects against influenza-associated bacterial pneumonia. (A) Study design with groups of C57BL/6 mice infected with 100 PFUs of influenza A PR/8/34 and receiving a single dose of FY1 or IgC control on days 1 to 5 after influenza infection. Mice received secondary infection with $5 \times 10^{7}$ CFUs of MRSA on day 6 after influenza, and samples were harvested 24 hours following bacterial infection $(n=8)$. (B) Bacterial colony counts in the lung. (C and $\mathbf{D})$ Weight loss on days 6 and 7 after influenza infection. (E) Survival curves of mice that received $\mathrm{FY} 1$ or IgG control on days 1,3 , or 5 after influenza infection. Mice received secondary infection with $5 \times 10^{8}$ CFUs of MRSA on day 6 after influenza $(n=12)$. ( $\mathbf{F}$ and $\mathbf{G})$ Total protein and IgM concentrations in the BALF. ${ }^{*} P<0.05$. Significance was tested by 1-way ANOVA using Dunnett's multiple comparison test except for $\mathbf{E}$ where a log-rank Mantel-Cox test was performed. Each experiment was independently performed twice, and data are shown from combined experiments. Data represent mean \pm SEM. 
A

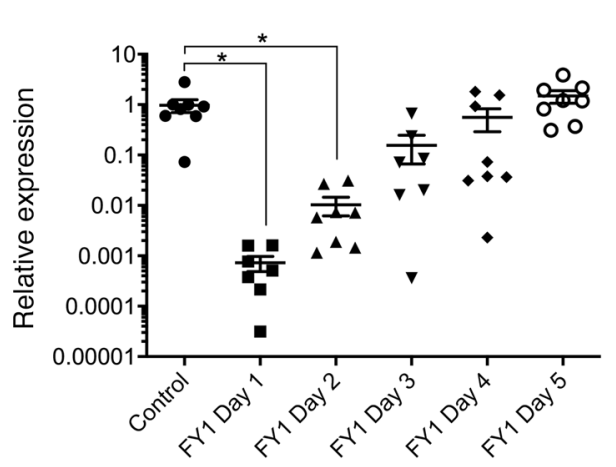

B

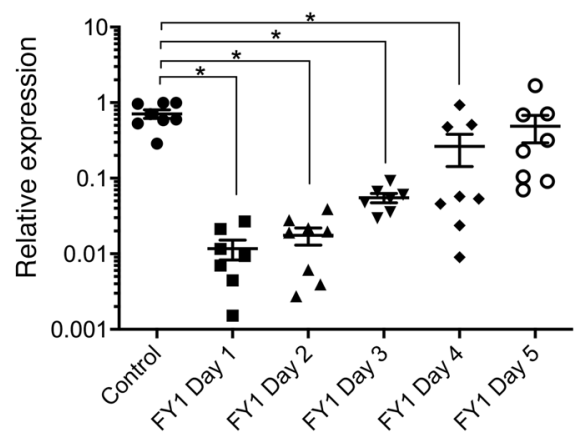

E

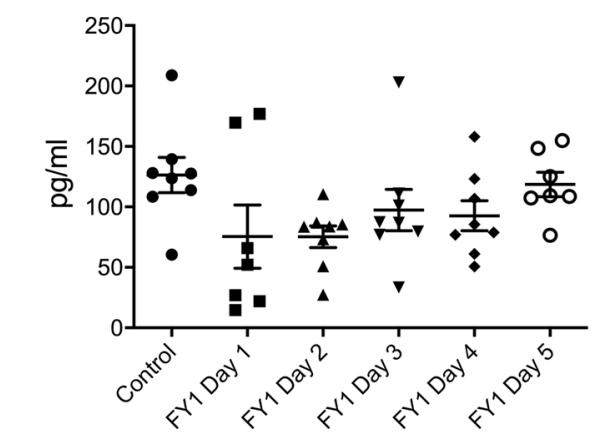

IFN $\gamma$
C

IFN $\beta$

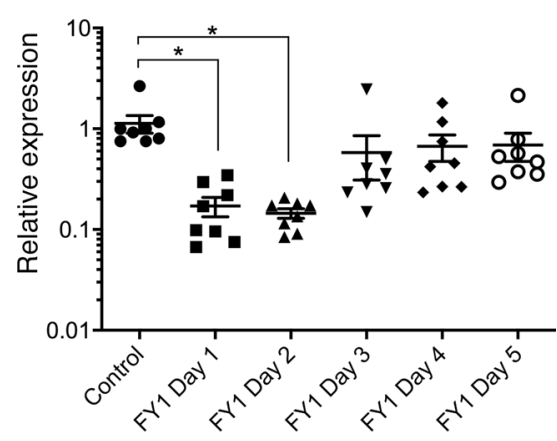

D

IFN $\lambda$

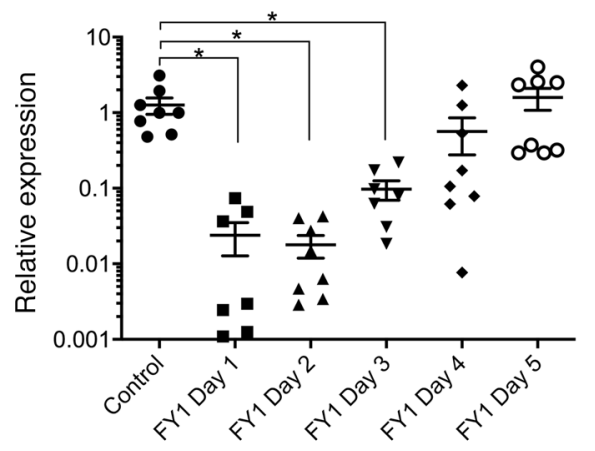

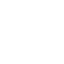

Figure 2. Influenza HA targeting mAb FY1 inhibits viral replication and subsequent IFN production during influenza-associated bacterial pneumonia. C57BL/6 mice infected with 100 PFUs of influenza A PR/8/34 and received a single dose of FY1 or IgG control on days 1 to 5 after influenza infection. Mice received secondary infection with $5 \times 10^{7}$ CFUs of MRSA on day 6 after influenza and samples were harvested 24 hours following bacterial infection ( $n=8$ ). (A) Influenza matrix protein expression in lung tissue measured by RT-PCR $(n=8)$. (B-D) IFN gene expression in lung tissue measured by RT-PCR ( $n=8$ ). (E) IFN protein concentrations in lung tissue measured by Bio-plex $(n=8) .{ }^{*} P<0.05$. Significance was tested by 1-way ANOVA using Dunnett's multiple comparison test. Each experiment was independently performed twice, and data are shown from combined experiments. Data represent mean \pm SEM.

S. aureus $\alpha$-toxin targeting $m A b$ MEDI4893* protects against influenza-associated bacterial pneumonia. A limitation of current human influenza therapeutics is the need to deliver drug (oseltamivir) during the first 48 hours of infection. In our murine model, we have shown that FY1 extends survival efficacy through day 3 after infection; however, therapeutics for later infection time points would be more desirable. FY1 induced inflammatory changes and protected against bacterial burden as late as 5 days after infection. The $S$. aureus $\alpha$-toxin targeting the mAb MEDI4893* was previously shown to decrease bacterial burden and improve survival during $S$. aureus pneumonia in mice (14). To examine the effect of MEDI4893* during influenza A and MRSA superinfection, C57BL/6 mice were challenged with influenza A PR/8/34 and then infected 6 days later with MRSA (USA300). We administered MEDI4893* on day 5 after influenza and 24 hours prior to $S$. aureus challenge (Figure 6A). Mice that received the $\alpha$-toxin targeting mAb showed increased survival compared with control mice (Figure 6B). In order to determine whether MEDI4893* could improve mortality in conjunction with anti-HA antibody during influenza and secondary MRSA pneumonia, we challenged mice with influenza A and MRSA as described and administered FY1 and MEDI4893* on day 5 after influenza (Figure 6C). We found the mice that received MEDI4893* in combination with FY1 had improved survival compared with the control group (Figure 6D). As reported in Figure 1, FY1 delivered 5 days after influenza infection did not alter survival rates. Notably, singular infection with either influenza or MRSA alone is not lethal (data not shown). These data suggest that combination therapy targeting both the virus and the bacteria may provide benefit at time points later during influenza infection than any currently available treatment.

\section{Discussion}

Our study demonstrates that specific pathogen-targeting antibodies can be used during influenza infection to protect against secondary bacterial pneumonia. The mAb FY1, shown previously to broadly neutralize 
A

Total cells

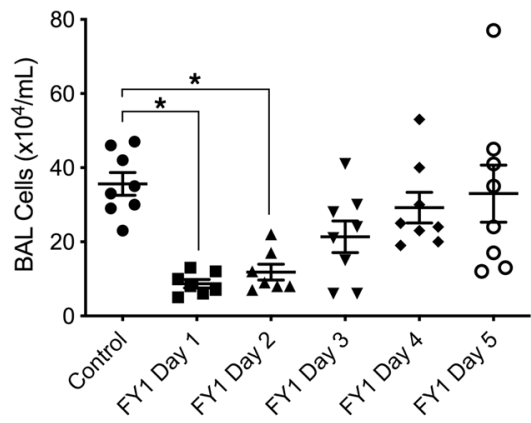

D

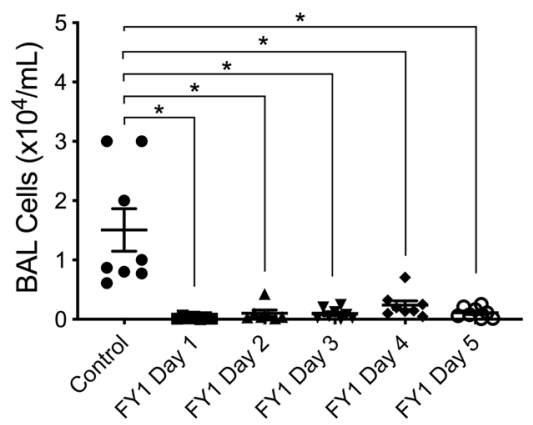

G

$$
\text { IL-10 }
$$

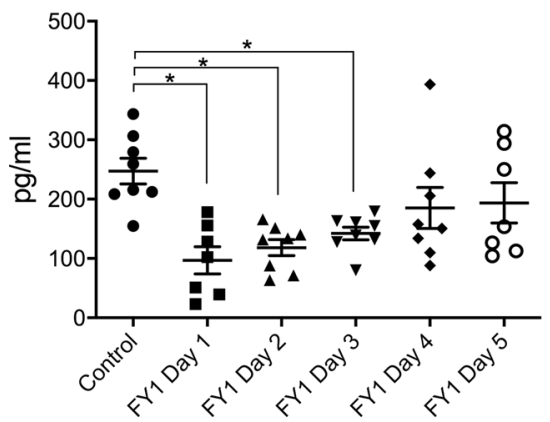

B

PMNs

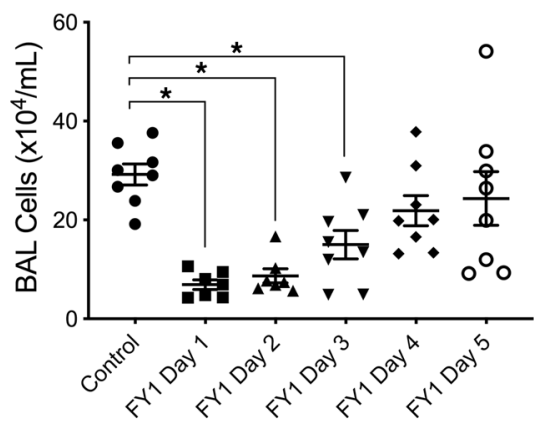

E

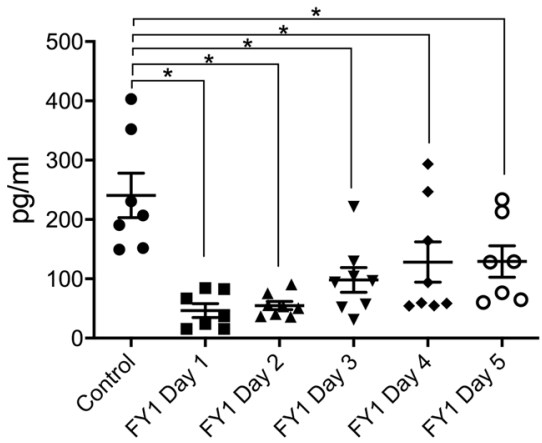

C

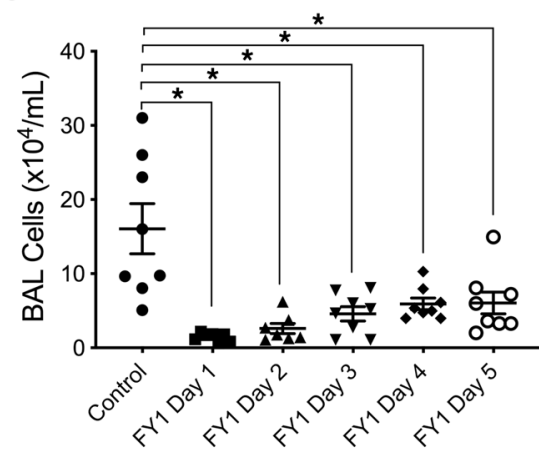

$F$

$\mathrm{KC}$

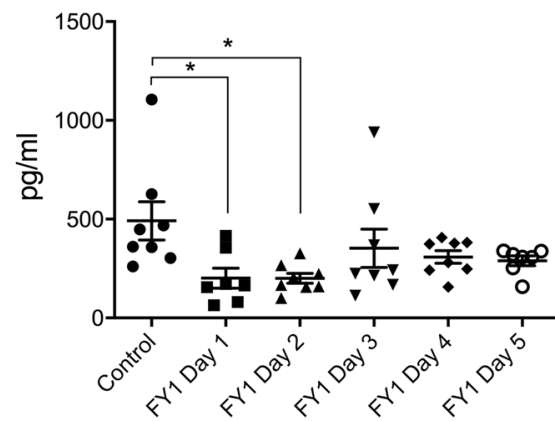

Figure 3. Influenza HA targeting mAb decreases airway inflammation and inhibits cytokine responses in the lung during influenza-associated bacterial pneumonia. C57BL/6 mice infected with $100 \mathrm{PFUs}$ of influenza A PR/8/34 received a single dose of FY1 or lgG control on days 1 to 5 after influenza infection. Mice received secondary infection with $5 \times 10^{7}$ CFUs of MRSA on day 6 after influenza and samples were harvested 24 hours following bacterial infection ( $n=8)$. (A) Bronchoalveolar lavage cell counts $(n=8)$. (B-D) Lavage cell differential counts $(n=8)$. (E-G) Cytokine and chemokine concentrations in lung tissue measured by Bio-plex $(n=8)$. ${ }^{*} P<0.05$. Significance was tested by 1 -way ANOVA using Dunnett's multiple comparison test. Each experiment was independently performed twice, and data are shown from combined experiments. Data represent mean \pm SEM.

influenza viruses, leads to decreased morbidity and mortality associated with secondary bacterial superinfections that commonly complicate influenza. Using a murine model of influenza and bacterial superinfection, we show that FY1 results in decreased bacterial burden of MRSA during superinfection. The use of FY1 decreases acute lung injury, decreases airway inflammation, and enhances macrophage function in the lung. FY1's reduction of influenza viral burden and acute lung injury likely leads to decreased bacterial burden during superinfection. Furthermore, the $S$. aureus $\alpha$-toxin-neutralizing mAb, MEDI4893*, increased survival when coadministered with FY1 late during superinfection.

Currently, neuraminidase inhibitors are recommended for the treatment and prevention of influenza infection. Clinical benefit is greatest when neuraminidase inhibitors are given within 48 hours of influenza-like symptom onset. Multiple meta-analyses (23-25) have been performed to assess the efficacy of neuraminidase inhibitors and report varied conclusions regarding both symptom management and prevention of complications such as secondary bacterial pneumonia. Although vaccines reduce the frequency of influ- 
A

$\mathrm{CD}_{11 \mathrm{c}^{+}}$

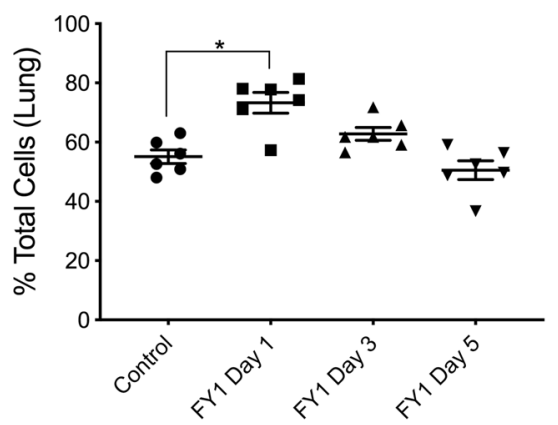

D

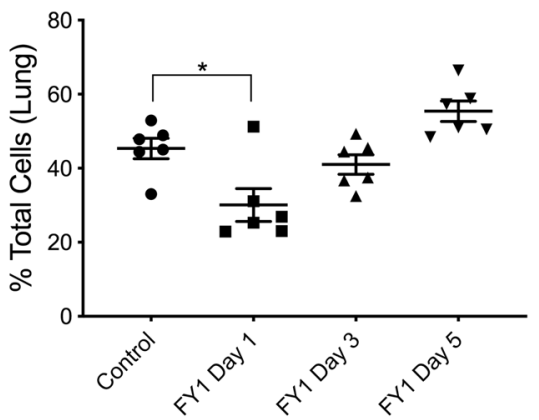

G

$\mathrm{CD}_{11 b^{+} \text {SiglecF}}{ }^{+}$Ly6G $^{-}$

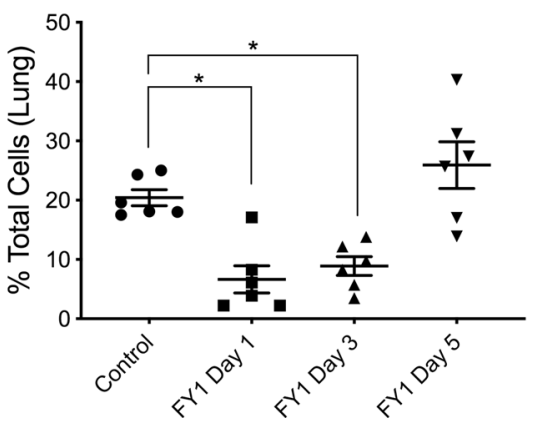

B

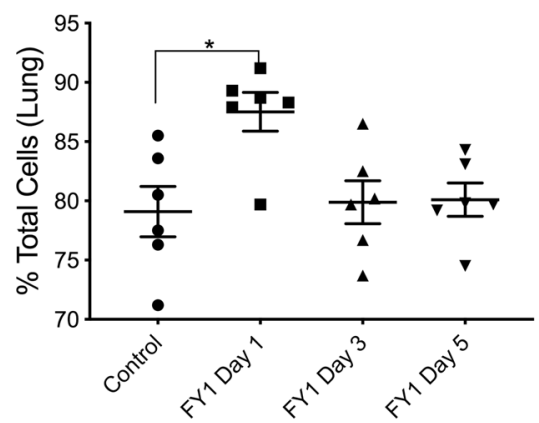

E

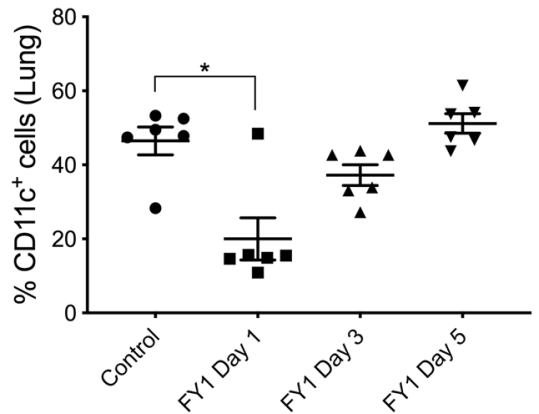

H

CD11b+ Ly6G $^{+}$

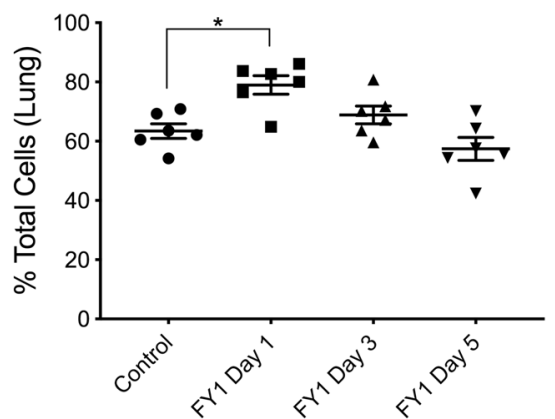

C

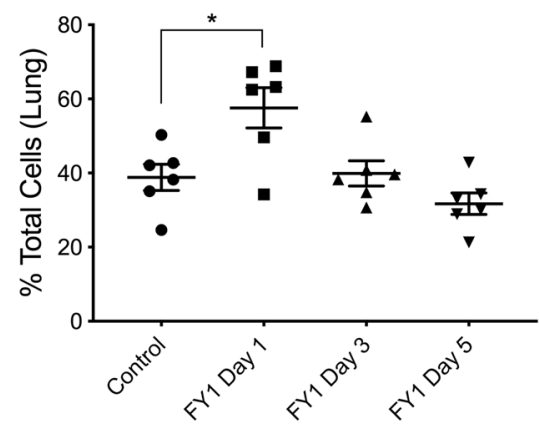

$\mathbf{F}$

\section{$\mathrm{CD}^{11 c^{-}} \mathrm{F} 480^{+}$SiglecF-}

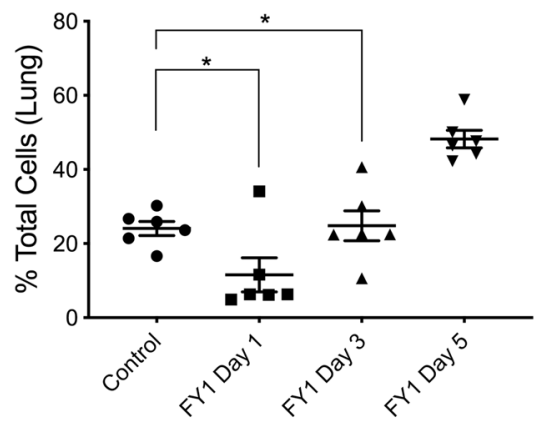

- Ly6G (lo)

I

Ly6G $^{+}$

o Ly6G (hi)

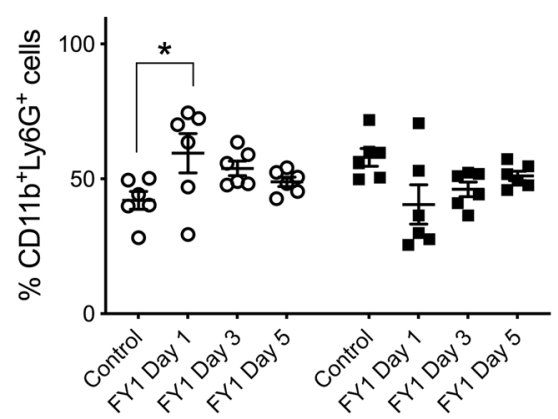

Figure 4. Influenza HA targeting mAb alters macrophage and neutrophil populations in the lung during influenza-associated bacterial pneumonia. C57BL/6 mice infected with 100 PFUs of influenza A PR/8/34 and received a single dose of FY1 on days 1, 3, or 5 after influenza infection. Mice received secondary infection with $5 \times 10^{7}$ CFUs of MRSA on day 6 after influenza and samples were harvested 24 hours following bacterial infection ( $n=6$ ). (A)

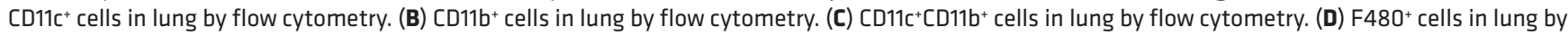

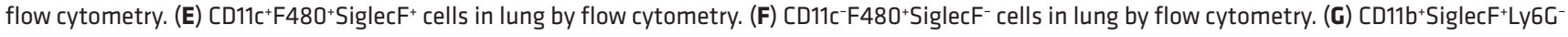

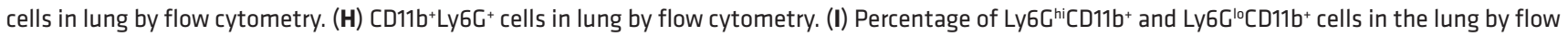
cytometry. ${ }^{*} P<0.05$. Significance was tested by 1 -way ANOVA using Dunnett's multiple comparison test. Data represent mean \pm SEM.

enza infection, vaccine efficacy varies widely due to mismatch between strain-specific vaccines, vaccine virus changes during egg-based production, and host factors such as age (1). Although additional research is underway to improve seasonal influenza vaccines, there is a shortage of effective methods to treat influenza infection and prevent secondary bacterial superinfection during influenza.

A related antibody, MEDI8852, has previously been shown to be useful in the prophylaxis and treatment of multiple types of influenza A viruses in mice. In addition, it decreases the transmission of influenza in other animal models (26). In our murine model of influenza and secondary bacterial pneumonia, we demonstrated that FY1 prevents morbidity, mortality, and decreases both viral and bacterial burden in the lung. Survival differences were seen in mice that received the influenza $\mathrm{mAb}$ on days 1 and 3 after influenza infection compared with mice that received the antibody on day 5 after influenza infection. The timing of the treatment 
A

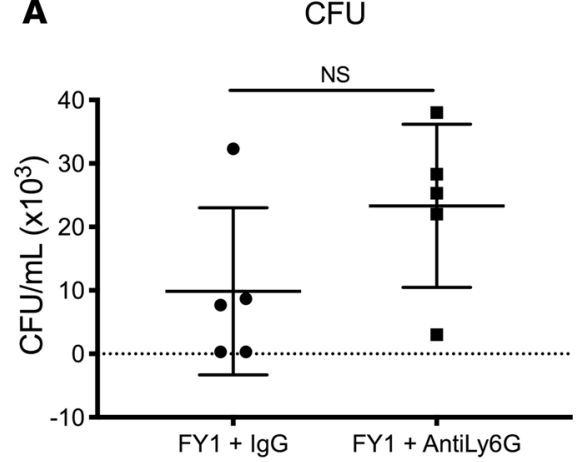

D

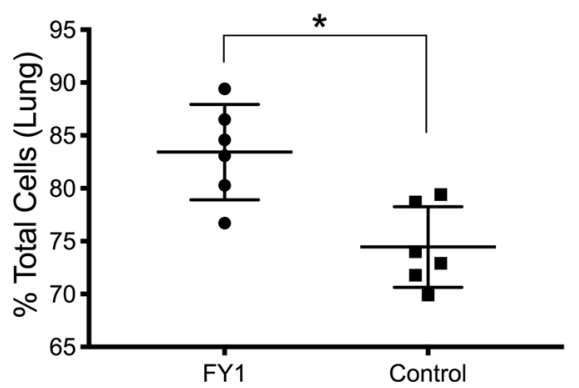

B

CD11b+Ly6g+ (uptake PMN)

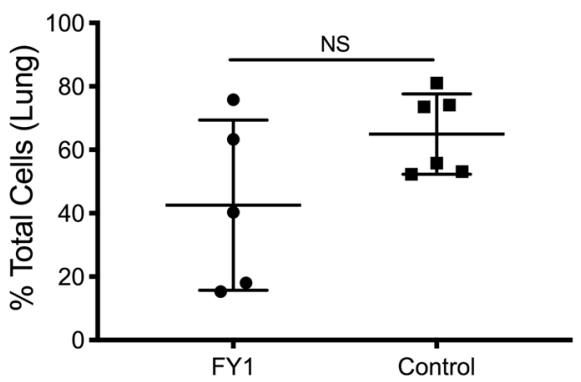

C

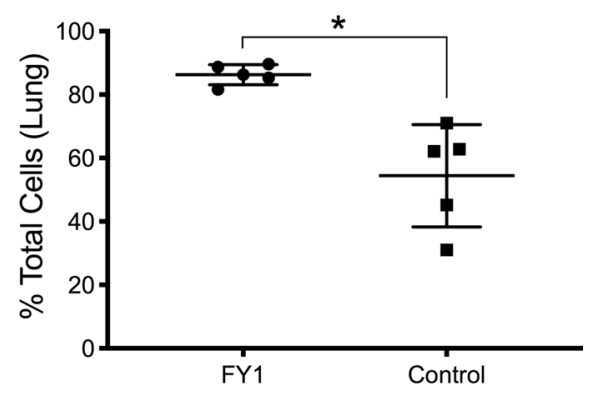

Figure 5. Neutrophils are not required for protection from influenza HA targeting $m A b$, but influenza HA targeting mAb enhances macrophage uptake of bacteria. C57BL/ 6 mice infected with 100 PFUs of influenza A PR/8/34 received a single dose of FY1 on day 1 after influenza infection. Mice received neutrophil depletion antibody $1 A 8$ (anti Ly6C) or IgG control at 48, 24, and 2 hours prior to secondary infection with $5 \times 10^{7}$ CFUs of MRSA on day 6 after influenza infection. Samples were harvested 24 hours following bacterial infection. (A) Bacterial colony counts in the lung $(n=6)$. C57BL/ 6 mice infected with 100 PFUs of influenza A PR/8/34 received a single dose of FY1 on day 1 after influenza infection. Mice received $5 \times 10^{7}$ CFUs of FITC-labeled MRSA on day 6 after influenza. Samples were harvested 24 hours following bacterial infection $(n=6)$. (B) Percentage of FITC+CD$11 b^{+}$Ly6C + cells in lung by flow cytometry. (C) Percentage of FITC+F $480^{+}$SiglecF+ cells in lung by flow cytometry. (D) Percentage of FITC+F480+SiglecF- cells in lung by flow cytometry. Significance was tested by unpaired $t$ test. Data represent mean \pm SEM.

administration is highly important and clinically relevant, as individuals need time to recognize influenza symptoms and present to a clinician. Although we recognize the inherent difference between humans and mouse modeling, administration of mAbs likely lengthens the time window for presentation of care compared with current treatments. Survival differences were only seen on days 1 and 3 after influenza infection administration, but bacterial burden was decreased when the $\mathrm{mAb}$ was given as late as 5 days after influenza infection. The downstream effects of decreased bacterial burden are not further elucidated but could potentially lead to decreased morbidity in patients who survive influenza complicated by bacterial superinfection. Furthermore, differences in alveolar capillary membrane permeability, a marker of acute lung injury, were seen in mice that received the influenza $\mathrm{mAb}$ at earlier time points after influenza infection (27). This finding indicates decreased injury from the initial influenza infection as a result of the use of the influenza $\mathrm{mAb}$ and is likely a mechanism for decreased susceptibility to bacterial superinfection in these mice.

Consistent with decreased viral burden, we demonstrated decreased levels of IFN- $\alpha$, IFN- $\beta$ and IFN- $\lambda$ in the lung when FY1 was administered at earlier time points after influenza infection. Prior studies have shown that dynamic changes in IFN signaling regulate susceptibility to postinfluenza MRSA pneumonia $(18,28)$. In our model, both IFN- $\alpha$ and $-\beta$ were decreased in the mice that received FY1 on days 1 and 2 after influenza infection, and expression increased as mice received the antibody later during the course of infection, corresponding to an increase in viral burden, similar to untreated animals. Interestingly, antibody treatment on day 3 after influenza infection did not significantly reduce viral burden but did still inhibit IFN production, bacterial burden, and mortality rates. Additional investigation is needed to determine if the improvement in mortality seen at days 1 and 3 after influenza infection is related to expression of a specific IFN. IFNs are known to induce chemoattractants during influenza infection $(29,30)$, and not surprisingly, we measured lower levels of most chemoattractants in mice that received the antibody at earlier time points. Although there were changes in chemoattractants, we did not see any differences in components of the type 17 immune pathway. This finding is noteworthy because we have previously shown that IL-17 and IL-1 play a role in promoting bacterial clearance during influenza infection $(16,22,31)$. We also investigated components of the type 2 immune pathway and did not measure any differences except for increased gene expression of IL-33 in mice that received FY1 earlier in the course of infection. We have previously shown that IL-33 recruits functional phagocytic neutrophils to the lung during postinfluenza MRSA pneumonia and that neutrophil depletion with IL-33 treatment led to increased susceptibility to MRSA pneumonia (22). We suspect that although IL-33 is increased in the mice that received FY1 earlier in the 
A

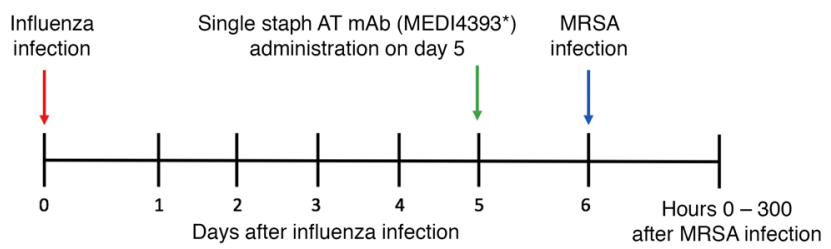

B

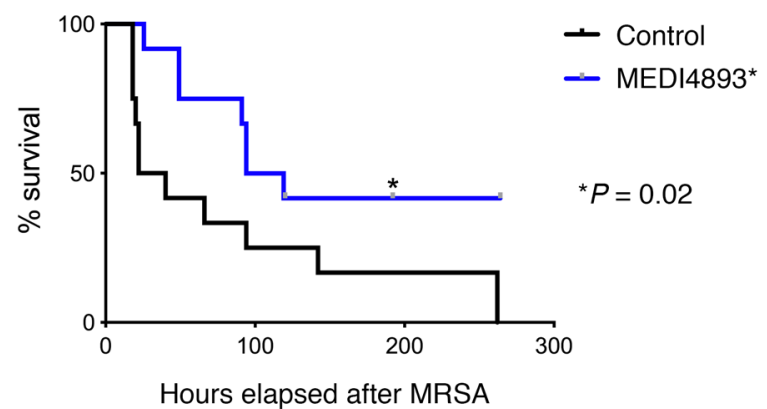

\section{C}

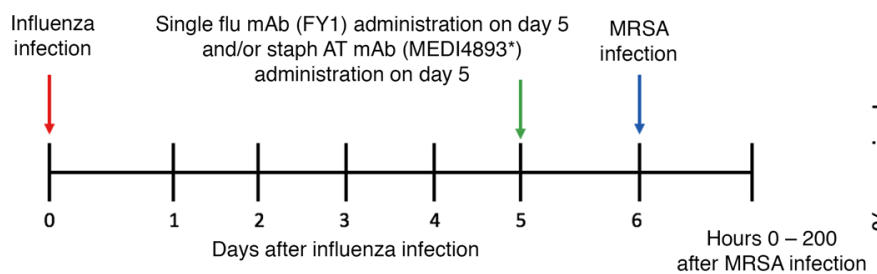

D

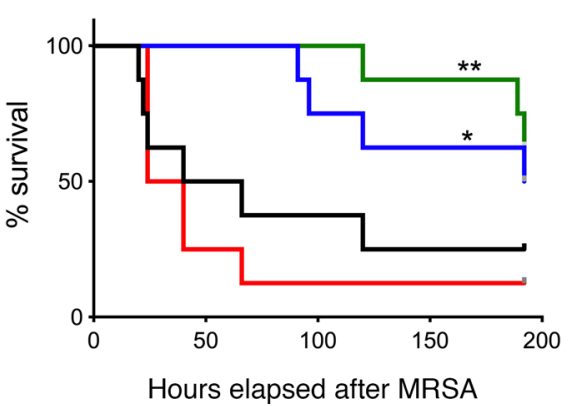

- Control

- MEDI4893*

- FY1

- MEDI4893*+FY1

${ }^{* *} P=0.002$

${ }^{*} P=0.05$

Figure 6. S. aureus $\alpha$-toxin targeting mAb MEDI4893* protects against influenza-associated bacterial pneumonia. (A) Study design with groups of C57BL/6 mice infected with 100 PFUs of influenza A PR/8/34 and receiving a single dose of MEDI4893* or lgG control on day 5 after influenza infection. Mice received secondary infection with $5 \times 10^{8}$ CFUs of MRSA on day 6 after influenza infection $(n=8)$. (B) Survival curve. (C) Study design with groups of C57BL/6 mice infected with 100 PFUs of influenza A PR/8/34 and receiving a single dose of MEDI4893* or FYI or IgG control or single doses of both MEDI4893* and FYI on day 5 after influenza infection. Mice received secondary infection with $5 \times 10^{8}$ CFUs of MRSA on day 6 after influenza infection ( $n$ = 12). (D) Survival curve. ${ }^{*} P$ as indicated in each experiment compared with control. Significance was tested by log-rank Mantel-Cox test. Each experiment was independently performed at least twice and data are shown from combined experiments.

course of influenza infection, it is likely not playing a role in the context of measuring any differences in other type 2 immune pathway cytokines. There is also no increased susceptibility to MRSA pneumonia in the mice that received FY1 in which neutrophils were depleted compared with mice that received FY1 and IgG control.

The lung consists of anatomic compartments that can be sampled by different methods (32). For example, in our studies, we collected both BALF from the airway compartment and whole lung tissue that is composed of interstitial tissue, vascular spaces, and small conducting airways and alveoli that we refer to as the lung compartment. Anatomic compartments all contribute to host defense against infection, but it is not well understood how leukocytes are recruited to compartments or migrate between compartments (32). Prior studies using ferrets infected with influenza H1N1 have investigated how lung compartments, designated as vascular, alveolar, and bronchiolar, differ in their response to infection and found that compartments have different histopathology, viral replication, and cytokine expression. These immune responses to influenza infection also vary in the same compartment at different time points following influenza challenge (33). In our current study, we demonstrated decreased numbers of leukocytes in the airway compartment when FY1 was given earlier in the course of infection. All inflammatory cells in the BALF were decreased including neutrophils, macrophages, and lymphocytes. Notably, decreased numbers of neutrophils in BALF are considered an important measurement of acute lung injury in animal models (27). In the lung compartment, we saw differential changes in percentages of specific cell populations. When FY1 was given earlier in the course of infection, we saw an increased percentage of $\mathrm{CD} 11 \mathrm{c}^{+}, \mathrm{CD} 11 \mathrm{~b}^{+}$, and $\mathrm{CD} 11 \mathrm{c}^{+} \mathrm{CD} 11 \mathrm{~b}^{+}$dendritic cell populations. Furthermore, we saw an increased percentage of dual-positive $\mathrm{CD} 11 \mathrm{~b}^{+} \mathrm{Ly}_{6 \mathrm{G}} \mathrm{G}^{+}$neutrophils in the lung. When FY1 was given earlier in the course of infection, there was a decreased proportion of $\mathrm{CD} 11 \mathrm{c}^{+} \mathrm{F} 480^{+}$SiglecF $^{+}$ (alveolar) and $\mathrm{CD}_{11} \mathrm{c}^{-} \mathrm{F} 480^{+}$SiglecF ${ }^{-}$(interstitial) macrophage populations. Neutrophils $\left(\mathrm{CD} 11 \mathrm{~b}^{+} \mathrm{Ly}_{6 \mathrm{G}}{ }^{+}\right.$) are phagocytes that help clear MRSA in the lungs, and neutrophil NADPH oxidase generates ROS necessary for 
antibacterial defense $(34,35)$. Influenza is known to impair NADPH oxidase-dependent bacterial clearance against MRSA in the setting of postinfluenza secondary bacterial superinfection (36). Our findings suggest that FY1 neutralizes influenza virus, allowing for increased recruitment of neutrophils to the lung during secondary MRSA pneumonia. Although we have previously shown that neutrophils are not required for bacterial clearance during postinfluenza bacterial pneumonia (17), neutrophils still remain a key mechanism by which bacteria are killed during pneumonia. We measured an increased percentage of $\mathrm{Ly}_{6} \mathrm{G}^{\mathrm{hi}} \mathrm{CD} 11 \mathrm{~b}^{+}$mature neutrophils in the mice that received FY1 earlier in the course of infection. In a pulmonary murine model of tularemia, survival differences have been associated with recruitment of either immature (Ly6C ${ }^{\text {hi }} \mathrm{Ly}_{6 \mathrm{G}}$ ) or mature (Ly6 $\mathrm{G}^{\text {hi}}$ Ly6 $\mathrm{C}^{\mathrm{int} / 10}$ ) neutrophils. In lethal tularemia infection, lungs are infiltrated by immature myeloid cells and myeloid cell death drives pathogenesis. In sublethal tularemia, mature myeloid cells are recruited and found to be protective (37). When we depleted neutrophils in mice that received FY1 early in the course of infection, however, we saw no differences in bacterial burden compared with mice that received FY1 alone. Therefore, although we measured an increased percentage of $\mathrm{Ly} 6 \mathrm{G}^{\mathrm{hi}} \mathrm{CD} 11 \mathrm{~b}^{+}$mature neutrophils in the mice that received FY1 earlier in the course of infection, neutrophils are not required for FY1-mediated protection. In addition, neutrophil uptake and binding of bacteria does not seen to be affected by FY1. During postinfluenza MRSA pneumonia, type I IFN signaling attenuates $\mathrm{Ly}_{6} \mathrm{G}^{+}$cell ability to kill MRSA on day 7 after influenza infection (28). Suppression of IFN- $\beta$ and IFN- $\lambda$ upon antibody treatment on day 3 after influenza infection herein is consistent with improved bacterial clearance and mortality. Although neutrophils are not required for FY1-mediated protection, FY1 enhances the function of both alveolar and interstitial macrophages to uptake and bind bacteria, suggesting that FY1 positively affects macrophage function in the lung. We also measured increased concentrations of proinflammatory mediators IL- 6 and KC in the lung in mice that received FY1 earlier in the course of infection, consistent with decreased acute lung injury (27). Notably, in our studies, we measured decreased neutrophils in BALF representing the airway compartment and increased neutrophils in whole lung tissue representing the lung compartment. This discrepancy is likely due to differences in the immune response within different anatomic compartments and the timing of our tissue harvest being a single time point and not necessarily reflecting changes in immune response kinetics over time.

In summary, we have demonstrated that FY1's reduction of influenza viral burden and acute lung injury likely leads to decreased mortality and decreased bacterial burden during superinfection. We have measured acute lung injury by multiple methods to show that FY1 reduces both alveolar capillary permeability and the inflammatory response to reduce acute lung injury during postinfluenza MRSA pneumonia in mice. We have also shown that FY1 enhances macrophage function in the lung and the ability of macrophages to uptake and bind bacteria during influenza infection. Currently, no vaccine exists to prevent infection with $S$. aureus. Approximately 30\% of individuals are colonized with $S$. aureus and nasopharyngeal colonization confers increased risk of disease. Strains such as MRSA are highly resistant to certain antibiotics (38). The mAb MEDI4893* has been developed against the $S$. aureus $\alpha$-toxin and passive immunization prevents $S$. aureus pneumonia in mice (14). In addition, prophylactic treatment with MEDI4893* increases survival and decreases bacterial burden in an immunocompromised mouse model of $S$. aureus pneumonia (14). In our study, we administered MEDI4893* to mice during influenza infection and then infected them with MRSA to determine if the $\alpha$-toxin antibody had the same effect during ongoing influenza infection. There was increased survival in the mice that received MEDI4893*, showing that MEDI4893* can be given during influenza infection and still protect against $S$. aureus pneumonia. This could potentially be related to decreased lung injury in these mice. We propose that MEDI4893* may be used in humans during influenza infection to prevent the development of secondary MRSA pneumonia. Furthermore, we showed that MEDI4893* protects against postinfluenza $S$. aureus pneumonia in combination with the influenza $\mathrm{mAb}$ FY1. Further investigation will have to be performed to evaluate preferential timing and optimal regimens of influenza and $S$. aureus $\alpha$-toxin mAbs during superinfection to determine if efficacy can be achieved. Overall, our current study shows that viral- and bacterial-specific mAbs can be used during influenza infection to protect against secondary bacterial pneumonia.

\section{Methods}

Animal studies. Six- to 8-week-old male WT C57BL/6 mice were purchased from Taconic Farms. Mice were maintained under pathogen-free conditions at the Children's Hospital of Pittsburgh (University of Pittsburgh Medical Center), and experiments were conducted with approval from the University of Pittsburgh Institutional Animal Care and Use Committee. All studies used age- and sex-matched mice. 
Murine bacterial and viral infections. MRSA (USA300; provided as a gift by Alice Prince, Columbia University, New York, New York, USA) was cultured as detailed by ATCC instructions in casein hydrolysate yeast extract containing modified medium for 18 hours to a stationary growth phase. Mice were inoculated with $5 \times 10^{7} \mathrm{CFU}$ of MRSA in $50-\mu \mathrm{L}$ sterile PBS by oropharyngeal aspiration. In survival studies, mice were inoculated with $5 \times 10^{8}$ CFUs of MRSA in $50-\mu \mathrm{L}$ sterile PBS by oropharyngeal aspiration. Influenza A PR/8/34 H1N1 was used to inoculate mice with $100 \mathrm{PFUs}$ of influenza (in $50-\mu \mathrm{L}$ sterile PBS) by oropharyngeal aspiration. Viral burden was determined by quantitative real-time RT-PCR on lung RNA for viral matrix protein as previously described (31). Mice were sequentially challenged with influenza for 6 days followed by infection with bacteria for an additional 24 hours. FY1 was administered i.p. $(15 \mathrm{mg} / \mathrm{kg})$ at indicated time points. MEDI4893* $(15 \mathrm{mg} / \mathrm{kg})$ was administered i.p. at indicated time points.

Analysis of lung inflammation. At the indicated time points, mouse lungs were lavaged with 1-mL sterile PBS for inflammatory cell differential counts. The cranial lobe of the right lung was homogenized in sterile PBS by mechanical grinding. The resulting lung homogenate was used for bacterial colony counting and cytokine analysis by Bio-plex Multiplex immunoassay (Bio-Rad). Middle and caudal lobes of the right lung were snap-frozen and homogenized under liquid nitrogen for RNA extraction by an RNA isolation kit (Agilent Technologies). RNA analysis was performed by standard RT-PCR using Assay on Demand TaqMan probes and primers (Applied Biosystems).

Analysis of alveolar capillary permeability. At the indicated time points, mouse lungs were lavaged with 1-mL sterile PBS. BALF was spun down and a pellet consisting of inflammatory cells was removed. Total protein was measured in the remaining BALF using the Coomassie Protein Assay Reagent Kit (Thermo Scientific) and total IgM was measured using the Mouse IgM Uncoated ELISA Kit (Invitrogen).

Depletion antibodies. Mice received $250 \mu \mathrm{g}$ of $1 \mathrm{~A} 8$ (anti-Ly6G) or IgG control from BioXcel in 200- $\mu \mathrm{L}$ sterile PBS by i.p. injection at 48,24 , and 2 hours prior to $S$. aureus challenge.

Flow cytometry. Whole mouse lungs were digested with collagenase as previously described (22). Cells were stained with fluorescent conjugated antibodies for F4/80, CD11b, CD11c, Ly6G, and Siglec-F (BD Biosciences). Staining was determined using the BD FACSAria machine, and data were analyzed using the FlowJo software package. Gating strategies are shown in Supplemental Figure 4.

S. aureus neutrophil and macrophage binding and uptake. S. aureus was labeled with FITC (Life Technologies). FITC-labeled $S$. aureus were instilled into mice, and lung tissue was harvested at 24 hours following bacterial challenge. Lung cells were stained with fluorescent conjugated antibodies against F4/80, CD11b, CD11c, Ly6G, and Siglec-F (BD Biosciences). Flow cytometry was performed to determine total percentage of FITC $^{+}$cells.

Statistics. All data are presented as the mean \pm SEM. Significance was tested by the unpaired $t$ test (for 2 means) or 1-way ANOVA (for multiple data groups), followed by the Tukey post hoc test. Significance of survival data was assessed by the Gehan-Breslow-Wilcoxon test. Data were analyzed using the GraphPad Prism software. $P<0.05$ was considered significant.

Study approval. Mouse experiments were conducted with approval from the University of Pittsburgh Institutional Animal Care and Use Committee, protocol 17071194.

\section{Author contributions}

KMR, KR, JFA, BRS, NLK, and TSC designed research studies. JFA provided reagents. KMR, KR, JMT, KLN, MJP, and KJM conducted experiments. KMR and KR acquired data. KMR, JFA, NLK, BRS, and TSC analyzed data and drafted the manuscript.

\section{Acknowledgments}

We acknowledge the National Heart, Lung, and Blood Institute for grant R01 HL107380 (to JFA), the Parker B. Francis Foundation (to KMR), and National Heart, Lung, and Blood Institute for grant K08 HL133445 (to KMR) and MedImmune. We also acknowledge Kevin J. McHugh for his technical support with our studies.

Address correspondence to: John F. Alcorn, Department of Pediatrics, Children's Hospital of Pittsburgh, 4401 Penn Avenue, 9127 Rangos, Pittsburgh, Pennsylvania 15224, USA. Phone: 412.692.7595; Email: john.alcorn@chp.edu. 
1. Paules CI, Sullivan SG, Subbarao K, Fauci AS. Chasing seasonal influenza - the need for a universal influenza vaccine. $N E n g l J$ Med. 2018;378(1):7-9.

2. Petrova VN, Russell CA. The evolution of seasonal influenza viruses. Nat Rev Microbiol. 2018;16(1):47-60.

3. Kallewaard NL, et al. Structure and function analysis of an antibody recognizing all influenza A subtypes. Cell. 2016;166(3):596-608.

4. Rice TW, et al. Critical illness from 2009 pandemic influenza A virus and bacterial coinfection in the United States. Crit Care Med. 2012;40(5):1487-1498.

5. Randolph AG, et al. Critically ill children during the 2009-2010 influenza pandemic in the United States. Pediatrics. 2011;128(6):e1450-e1458.

6. Palacios G, et al. Streptococcus pneumoniae coinfection is correlated with the severity of H1N1 pandemic influenza. PLoS One. 2009;4(12):e8540.

7. Morens DM, Taubenberger JK, Fauci AS. Predominant role of bacterial pneumonia as a cause of death in pandemic influenza: implications for pandemic influenza preparedness. J Infect Dis. 2008;198(7):962-970.

8. Nguyen AM, Noymer A. Influenza mortality in the United States, 2009 pandemic: burden, timing and age distribution. PLoS One. 2013;8(5):e64198.

9. Wong KK, et al. Influenza-associated pediatric deaths in the United States, 2004-2012. Pediatrics. 2013;132(5):796-804.

10. Finelli L, et al. Influenza-associated pediatric mortality in the United States: increase of Staphylococcus aureus coinfection. Pediatrics. 2008;122(4):805-811.

11. McCullers JA. The co-pathogenesis of influenza viruses with bacteria in the lung. Nat Rev Microbiol. 2014;12(4):252-262

12. Iverson AR, Boyd KL, McAuley JL, Plano LR, Hart ME, McCullers JA. Influenza virus primes mice for pneumonia from Staphylococcus aureus. J Infect Dis. 2011;203(6):880-888.

13. Robinson KM, Kolls JK, Alcorn JF. The immunology of influenza virus-associated bacterial pneumonia. Curr Opin Immunol. 2015;34:59-67.

14. Hua L, et al. MEDI4893* promotes survival and extends the antibiotic treatment window in a Staphylococcus aureus immunocompromised pneumonia model. Antimicrob Agents Chemother. 2015;59(8):4526-4532.

15. Oganesyan V, et al. Mechanisms of neutralization of a human anti- $\alpha$-toxin antibody. J Biol Chem. 2014;289(43):29874-29880.

16. Kudva A, et al. Influenza A inhibits Th17-mediated host defense against bacterial pneumonia in mice. J Immunol. 2011;186(3):1666-1674.

17. Robinson KM, et al. Influenza A virus exacerbates Staphylococcus aureus pneumonia in mice by attenuating antimicrobial peptide production. J Infect Dis. 2014;209(6):865-875.

18. Planet PJ, et al. Lambda interferon restructures the nasal microbiome and increases susceptibility to staphylococcus aureus superinfection. MBio. 2016;7(1):e01939-e01915.

19. Parker D, Planet PJ, Soong G, Narechania A, Prince A. Induction of type I interferon signaling determines the relative pathogenicity of Staphylococcus aureus strains. PLoS Pathog. 2014;10(2):e1003951.

20. Ghoneim HE, Thomas PG, McCullers JA. Depletion of alveolar macrophages during influenza infection facilitates bacterial superinfections. J Immunol. 2013;191(3):1250-1259.

21. Damjanovic D, Lai R, Jeyanathan M, Hogaboam CM, Xing Z. Marked improvement of severe lung immunopathology by influenza-associated pneumococcal superinfection requires the control of both bacterial replication and host immune responses. Am J Pathol. 2013;183(3):868-880.

22. Robinson KM, Ramanan K, Clay ME, McHugh KJ, Rich HE, Alcorn JF. Novel protective mechanism for interleukin-33 at the mucosal barrier during influenza-associated bacterial superinfection. Mucosal Immunol. 2018;11(1):199-208.

23. Ebell MH, Call M, Shinholser J. Effectiveness of oseltamivir in adults: a meta-analysis of published and unpublished clinical trials. Fam Pract. 2013;30(2):125-133.

24. Jefferson T, et al. Neuraminidase inhibitors for preventing and treating influenza in healthy adults and children. Cochrane Database Syst Rev. 2014;(4):CD008965.

25. Dobson J, Whitley RJ, Pocock S, Monto AS. Oseltamivir treatment for influenza in adults: a meta-analysis of randomised controlled trials. Lancet. 2015;385(9979):1729-1737.

26. Paules CI, et al. The hemagglutinin A stem antibody MEDI8852 prevents and controls disease and limits transmission of pandemic influenza viruses. J Infect Dis. 2017;216(3):356-365

27. Matute-Bello G, et al. An official American Thoracic Society workshop report: features and measurements of experimental acute lung injury in animals. Am J Respir Cell Mol Biol. 2011;44(5):725-738.

28. Shepardson KM, et al. Differential type I interferon signaling is a master regulator of susceptibility to postinfluenza bacterial superinfection. MBio. 2016;7(3):e00506-16.

29. Veckman V, Osterlund P, Fagerlund R, Melén K, Matikainen S, Julkunen I. TNF- $\alpha$ and IFN- $\alpha$ enhance influenza-A-virus-induced chemokine gene expression in human A549 lung epithelial cells. Virology. 2006;345(1):96-104.

30. Oslund KL, Baumgarth N. Influenza-induced innate immunity: regulators of viral replication, respiratory tract pathology \& adaptive immunity. Future Virol. 2011;6(8):951-962.

31. Robinson KM, et al. Influenza A exacerbates Staphylococcus aureus pneumonia by attenuating IL-1 $\beta$ production in mice. $J$ Immunol. 2013;191(10):5153-5159.

32. Tschernig T, Pabst R. What is the clinical relevance of different lung compartments? BMC Pulm Med. 2009;9:39

33. Vidaña B, et al. Involvement of the different lung compartments in the pathogenesis of pH1N1 influenza virus infection in fer rets. Vet Res. 2016;47(1):113.

34. Köhler J, et al. NADPH-oxidase but not inducible nitric oxide synthase contributes to resistance in a murine Staphylococcus aureus Newman pneumonia model. Microbes Infect. 2011;13(11):914-922.

35. Jann NJ, Schmaler M, Ferracin F, Landmann R. TLR2 enhances NADPH oxidase activity and killing of Staphylococcus aureus by PMN. Immunol Lett. 2011;135(1-2):17-23.

36. Sun K, Metzger DW. Influenza infection suppresses NADPH oxidase-dependent phagocytic bacterial clearance and enhances susceptibility to secondary methicillin-resistant Staphylococcus aureus infection. J Immunol. 2014;192(7):3301-3307. 
37. Periasamy S, Avram D, McCabe A, MacNamara KC, Sellati TJ, Harton JA. An immature myeloid/myeloid-suppressor cell response associated with necrotizing inflammation mediates lethal pulmonary tularemia. PLoS Pathog. 2016;12(3):e1005517.

38. Giersing BK, Dastgheyb SS, Modjarrad K, Moorthy V. Status of vaccine research and development of vaccines for Staphylococcus aureus. Vaccine. 2016;34(26):2962-2966. 